15

\title{
Линейка высокочастотных спектрометров электронного парамагнитного резонанса с микроволновым и оптическим каналами регистрации
}

\author{
(C) Р.А. Бабунц, А.Г. Бадалян, А.С. Гурин, Б.Р. Намозов, \\ Н.Г. Романов, П.Г. Баранов
}

Физико-технический институт им. А.Ф. Иофффе РАН, Санкт-Петербург

E-mail: roman.babunts@gmail.com

Поступило в Редакцию 8 сентября 2016 г.

Описана конструкция линейки высокочастотных спектрометров электронного парамагнитного резонанса/оптически детектируемого магнитного резонанса, работающих в непрерывном и импульсном режимах в диапазонах длин волн 2,3 , 4 и $8 \mathrm{~mm}$. Регистрация сигналов производится как по микроволновому, так и по оптическому каналам. Линейка спектрометров выполнена по единому принципу.

DOI: 10.21883/PJTF.2017.08.44536.16475

Электронный парамагнитный резонанс (ЭПР) и основанные на ЭПР методики двойных резонансов - оптически детектируемый магнитный резонанс (ОДМР) и двойной электронно-ядерный резонанс (ДЭЯР) являются наиболее информативными аналитическими методами изучения свойств конденсированных материалов и наноструктур в физике, химии, биологии, медицине. Основной мировой тенденцией развития приборной базы этих методов является переход к более высоким частотам и более сильным магнитным полям. При этом увеличивается чувствительность метода и одновременно уменьшается объем исследуемого образца. Минимально регистрируемая концентрация спинов $\left(N_{\min } / V_{S}\right) \propto \omega^{-3 / 2}$ в случае, если объем образца $\left(V_{S}\right)$ изменяется пропорционально объему резонатора спектрометра $V_{C}$, тогда как минимальное число регистрируемых спинов $N_{\min } \propto \omega^{-9 / 2}$, когда объем образца постоянный (для образцов малых размеров). Увеличение рабочей частоты требует работы с более сильными магнитными полями. При этом увеличивается разрешение по $g$-факторам, а использова- 
ние более сильного магнитного поля ведет к увеличению фактора Больцмана, который, помимо повышения играет важную роль во многих спин-зависимых процессах, включая динамическую поляризацию ядер. С увеличением частоты расширяются возможности исследования высокоспиновых систем, в которых имеются большие расщепления тонкой структуры. В сильном магнитном поле для таких центров упрощается идентификация их спектров из-за уменьшения эффектов второго порядка.

Переход к технике ОДМР, в которой магнитный резонанс регистрируется по изменению поглощения или излучения света, приводит к огромному повышению чувствительности, вплоть до возможности регистрировать одиночный спин, пространственной и спектральной селективности. В методе высокочастотного ДЭЯР преимущество высоких магнитных полей проявляется в лучшем разрешении ларморовских частот различных ядер. Благодаря более короткому времени звона резонатора $\left(T_{C}\right)$, которое обратно пропорционально частоте $T_{C} \propto \omega^{-1}[1]$, существенно повышается чувствительность в импульсных методах высокочастотного ЭПР.

Спектры ЭПР в общем виде описываются спиновым гамильтониаHOM [2]

$$
H=\mu_{B} \cdot \mathbf{B} \cdot \mathbf{g} \cdot \mathbf{S}+\mathbf{S} \cdot \mathbf{D} \cdot \mathbf{S}+\mathbf{S} \cdot \mathbf{A} \cdot \mathbf{I}-g_{I} \cdot \mu_{N} \cdot \mathbf{B} \cdot \mathbf{I}+\mathbf{I} \cdot \mathbf{Q} \cdot \mathbf{I},
$$

где первый член представляет собой зеемановское взаимодействие для электронного спина; второй и третий - расщепление тонкой структуры и сверхтонкое взаимодействие, а четвертый и пятый ядерные зеемановское и квадрупольное взаимодействия. Только первый и четвертый члены спинового гамильтониана (1) зависят от магнитного поля, поэтому исследование ЭПР на разных частотах позволяет разделить эти взаимодействия и корректно описать парамагнитные центры.

Обычные спектрометры ЭПР в качестве источников микроволнового излучения используют клистрон (в старых спектрометрах) или генератор на диоде Ганна, частота которых может перестраиваться и согласовываться с частотой рабочего резонатора. Преимуществом таких спектрометров является простота настройки, а недостатком сравнительно низкая стабильность частоты.

В работе [3] описан высокочастотный спектрометр ЭПР 3-mm диапазона, работающий на фиксированной частоте $94.9 \mathrm{GHz}$. В этом 
спектрометре используется импульсная схема регистрации ЭПР, причем настройка резонатора осуществляется по изменению амплитуды и фазы последовательности импульсов спинового эха. Недостатком такого спектрометра является отсутствие возможности визуально контролировать настройку рабочего резонатора при работе в непрерывном режиме.

При выборе частотных диапазонов линейки высокочастотных спектрометров учитывалось то, что с увеличением рабочей частоты спектрометров ЭПР выше $150 \mathrm{GHz}$ конструкция спектрометра значительно усложняется. При таких высоких частотах необходимо использовать сверхпроводящие магниты с напряженностью поля выше $11 \mathrm{~T}$. Такие магниты выполняются в виде соленоидов, тогда как магниты до $11 \mathrm{~T}$ выполняются в виде колец Гельмгольца. Магнит в виде колец Гельмгольца позволяет оперативно менять образцы, вращать их вокруг вертикальной оси для измерения угловых зависимостей спектров и использовать оптический канал. В спектрометре ЭПР 1-mm диапазона $(275 \mathrm{GHz})[4,5]$ применяется квазиоптический микроволновый блок, который не совместим с традиционными волноводными мостами для более низких частот.

Нами были выбраны диапазоны длин волн $2 \mathrm{~mm}$ (D-band), $3 \mathrm{~mm}$ (W-band), $4 \mathrm{~mm}$ (V-band) и $8 \mathrm{~mm}$ (Q-band), как наиболее удобные для линейки спектрометров ЭПР/ОДМР, в которой используется набор микроволновых блоков, выполненных по единому образцу.

Блок-схема спектрометра представлена на рис. 1. Общая для всей линейки магнитооптическая криогенная система выполнена на базе стандартного магнитооптического криостата 1 с диапазоном перестройки магнитных полей 0-7 Т, достаточным для работы на всех выбранных частотах. Резонатор с образцом размещается в изолированном объеме криостата, в котором температура регулируется в диапазоне от 1.5 до $300 \mathrm{~K}$. В использованном нами магнитооптическом криостате замкнутого цикла диаметр „теплого объема“ составлял $30 \mathrm{~mm}$. Горизонтальное направление магнитного поля обеспечивает возможность оперативной смены образцов и вращение образца вокруг вертикальной оси для измерения угловых зависимостей спектров.

Образец находится в микроволновом резонаторе. Разработана конструкция перестраиваемых одномодовых цилиндрических резонаторов $H_{011}$, которые для разных диапазонов отличаются только размерами. Связь осуществляется по боковой стенке, а частота перестраивается перемещением верхней крышки. Для сокращения потерь транспорти-

5 Письма в ЖТФ, 2017, том 43, вып. 8 


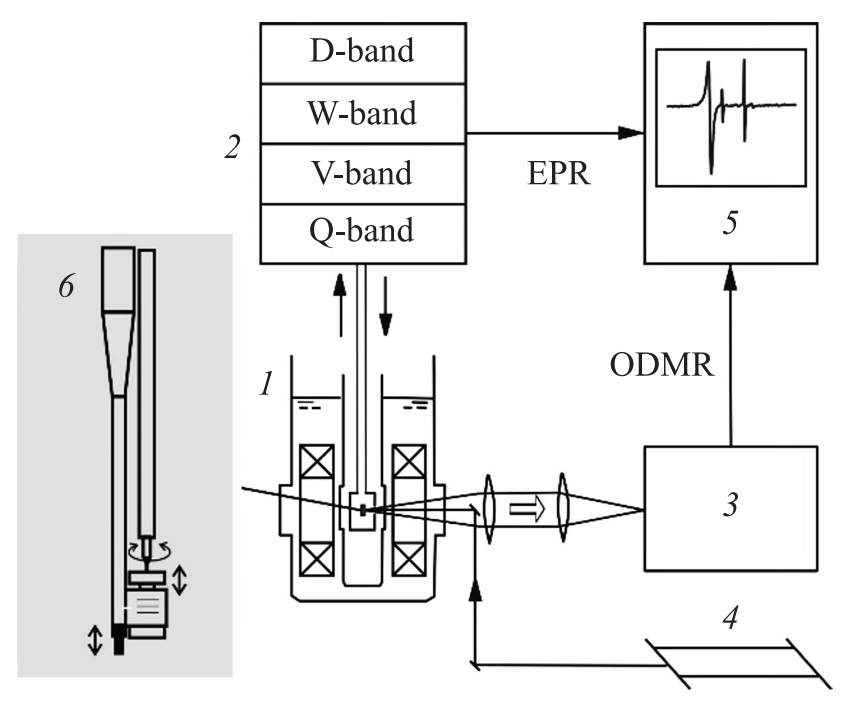

Рис. 1. Блок-схема линейки спектрометров ЭПР/ОДМР: 1 - криомагнитная система, 2 - микроволновые блоки, 3 - оптическая система регистрации, 4 - лазер, 5 - компьютер, 6 - вариант подключения рабочего резонатора.

ровка микроволной мощности от микроволнового блока к резонатору для всех рабочих диапазонов осуществляется по 8-mm волноводам с использованием переходных секций на волновод соответствующего диапазона. Регистрация ЭПР осуществляется как по отраженной от резонатора СВЧ-мощности (ЭПР), так и по изменению оптических характеристик образца (ОДМР). На рис. 1 показан пример регистрации ОДМР по интенсивности фотолюминесценции, регистрируемой фотоприемником 3 и возбуждаемой лазером 4. В экспериментах по ОДМР возможно также применение квазиоптической схемы подачи микроволновой мощности на образец, описанной в [6].

Микроволновые блоки построены по общей схеме и могут заменяться в зависимости от условий эксперимента. Упрощенная блоксхема микроволнового блока представлена на рис. 2. В схеме используется высокостабильный твердотельный генератор 1 фиксированной частоты $f_{1}$ (около $7 \mathrm{GHz}$ ), сигнал с которого поступает на умно-

Письма в ЖТФ, 2017, том 43, вып. 8 


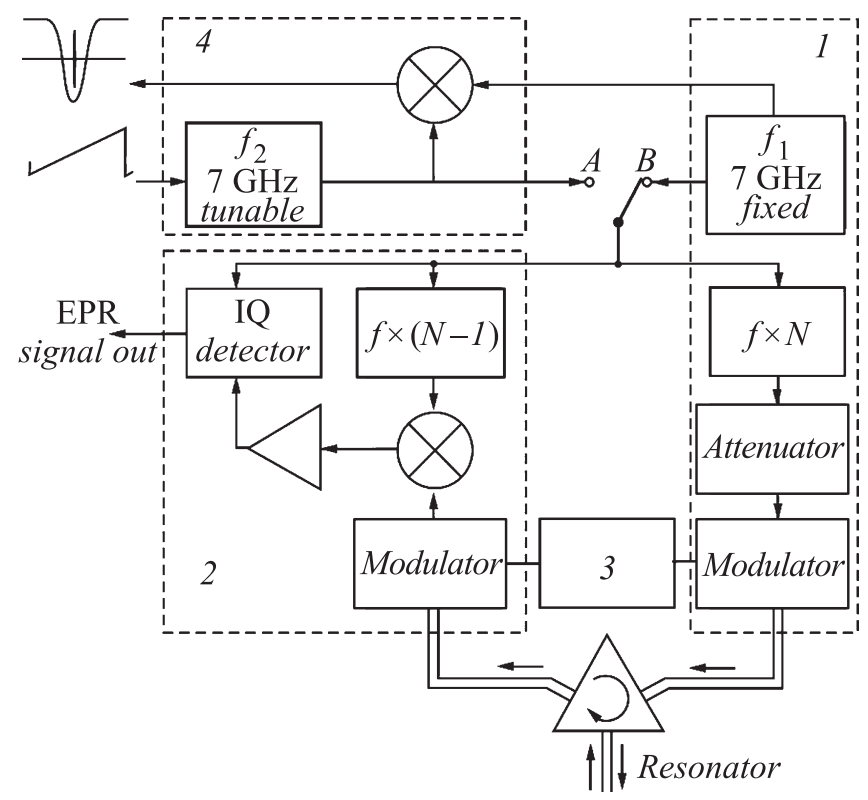

Рис. 2. Унифицированная блок-схема микроволнового блока: 1 - генератор фиксированной частоты, 2 - приемник, 3 - формирователь импульсов, $4-$ формирователь метки частоты.

жители частоты на $N$ (основной генератор) и на $N-1$ (гетеродин). Высокочастотный сигнал после усиления и прохождения аттенюатора через циркулятор поступает на резонатор, а отраженный от резонатора сигнал - на смеситель приемника 2, и после усилителя промежуточной частоты - на квадратурный (IQ) детектор. Коэффициент $N$ зависит от рабочей частоты. Например, для 2-mm диапазона $N=20$, для 3-mm диапазона $N=13$ и т.д. Модуляторы передатчика и приемника необходимы для работы спектрометра в импульсном режиме, например, при регистрации электронного спинового эха. Работой модуляторов управляет формирователь импульсов 3 , который создает необходимую последовательность импульсов и запрещает включение приемника при включенном передатчике. Длительность импульса (от $10 \mathrm{~ns}$ до $0.1 \mathrm{~s}$ ) задается с шагом $3.2 \mathrm{~ns}$.

$5^{*}$ Письма в ЖТФ, 2017, том 43, вып. 8 

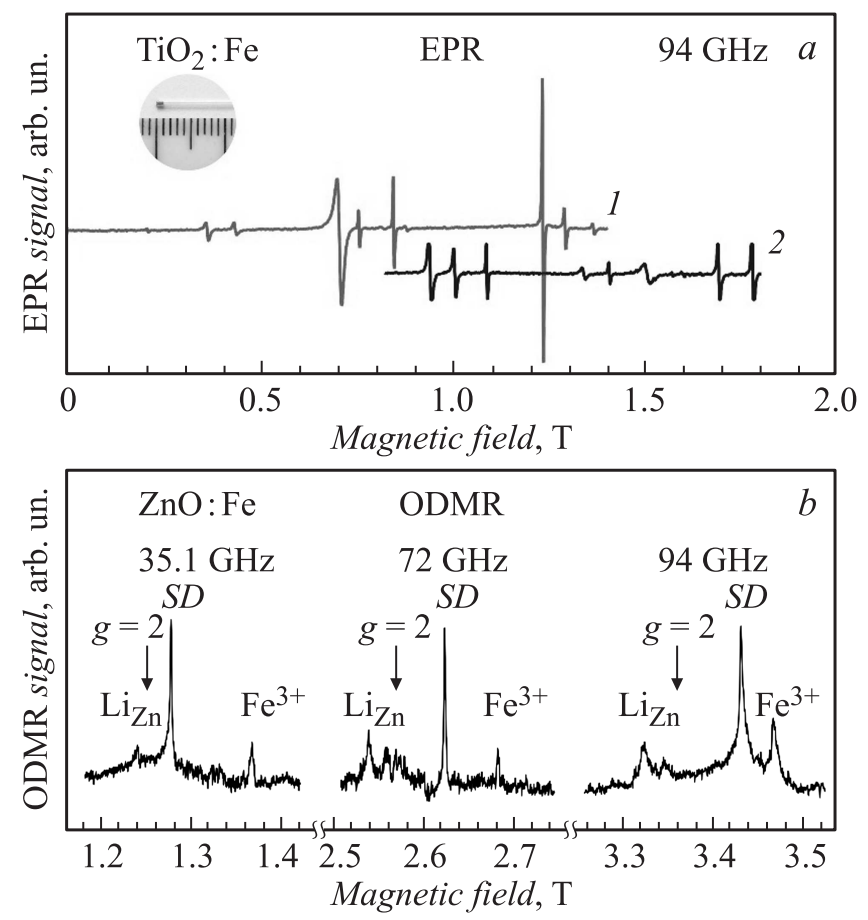

Рис. 3. $a-$ спектры ЭПР кристалла $\mathrm{TiO}_{2}: \mathrm{Fe}$, зарегистрированные при комнатной температуре на частоте $94 \mathrm{GHz}$ в одном и том же образце (показан на вставке) с использованием описанного в настоящей работе спектрометра 1 и спектрометра фирмы Bruker 2. $b$ - спектры ОДМР кристалла $\mathrm{ZnO}: \mathrm{Fe}$, зарегистрированные на частотах 35.1, 72 и $94 \mathrm{GHz}$ по интенсивности фотолюминесценции на длине волны $720 \mathrm{~nm}$ с применением микроволновых блоков: 8,4 и $3 \mathrm{~mm} . T=2 \mathrm{~K}$, ориентация кристалла $B \perp c$.

Задача визуальной настройки резонатора решается применением вспомогательного генератора 4 , частота которого $f_{2}$ управляется напряжением. В режиме настройки переключатель генераторов находится в положении „А“, что позволяет наблюдать на осциллографе резонансную кривую рабочего резонатора и сигнал биений двух генераторов („метку“), который формируется на высокочастотном смесителе в

Письма в ЖТФ, 2017, том 43, вып. 8 
момент совпадения с частотой основного генератора. Перестройка рабочего резонатора позволяет настроить его частоту на „метку“, т. е. на частоту рабочего генератора [7]. После настройки резонатора производится переключение в режим измерений (положение „, $\left.B^{“ 6}\right)$, в котором используется только высокостабильный генератор с фиксированной частотой.

Проведены контрольные измерения спектров ЭПР и ОДМР. На рис. 3, $a$ приведен спектр ЭПР ионов $\mathrm{Fe}^{3+}$ в кристалле $\mathrm{TiO}_{2}$, зарегистрированный на частоте $94 \mathrm{GHz}$ при комнатной температуре. Для сравнения показан спектр ЭПР того же образца, измеренный в аналогичных условиях на стандартном спектрометре фирмы Bruker. Отношение сигнал/шум примерно одинаково, что свидетельствует о высокой чувствительности разработанного нами спектрометра. Различие в положении линий ЭПР обусловлено различием ориентации кристалла в магнитном поле. Работа в режиме ОДМР иллюстрируется рис. $3, b$, на котором показаны спектры, зарегистрированные при $1.8 \mathrm{~K}$ по интенсивности фотолюминесценции кристаллов $\mathrm{ZnO}: \mathrm{Fe}$ с использованием микроволновых блоков 8,4 и $3 \mathrm{~mm}$. Видно, что смещение линий ОДМР мелких доноров (SD) и глубоких акцепторов $\operatorname{Li}_{\mathrm{Zn}}(S=1 / 2$, $g>2$ соответственно) относительно полей, соответствующих $g=2$, увеличивается пропорционально частоте, тогда как смещение сигнала ОДМР $\mathrm{Fe}^{3+}\left(S=5 / 2, g=2, D=-595 \cdot 10^{-4} \mathrm{~cm}^{-1}\right)$ относительно $g=2$ остается постоянным.

Таким образом, разработана линейка спектрометров ЭПР/ОДМР. Показана необходимость использования различных микроволновых частот для всестороннего исследования картины спиновых взаимодействий парамагнитных центров в изучаемом материале. Исследования могут производиться как в непрерывном, так и в импульсном режимах, а также с использованием оптической регистрации. Линейка спектрометров состоит из: стандартного магнитооптического криостата, набора микроволновых блоков на частоты 130, 95, 70 и $35 \mathrm{GHz}$, сконструированных по единой схеме и использующих единый принцип визуальной настройки резонатора и компьютерной системы управления и регистрации сигнала, для которой разработана специальная программа.

Работа была поддержана Российским научным фондом, грант № 1412-00859.

Письма в ЖТФ, 2017, том 43, вып. 8 


\section{Список литературы}

[1] Abragam A., Bleaney B. Electron Paramagnetic Resonance of Transition Ions. Oxford: Oxford University Press, 1970.

[2] Möbius K., Savitsky A. // High-Field EPR Spectroscopy on Proteins and their Model Systems: Characterization of Transient Paramagnetic States. Published by the Royal Society of Chemistry, 2008.

[3] Disselhorst J.A.J.M., van der Meer H.J., Poluektov O.G., Schmidt J. // Magn. Reson. Ser. A. 1995. V. 115. P. 183-188.

[4] Blok H., Disselhorst J.A.J.M., Orlinskii S.B. et al. // Physica B. 2003. V. 340-342. P. 1147-1150.

[5] Blok H., Disselhorst J.A.J.M., Orlinskii S.B., Schmidt J. // Magn. Reson. 2004. V. 166. P. 92-99.

[6] Бабуни, Р.А., Бадалян А.Г., Романов Н.Г. и др. // Письма в ЖТФ. 2012. Т. 38. B. 19 . C. $37-43$.

[7] Баранов П.Г., Бабуни Р.А., Бадалян А.Г., Романов Н.Г., Богданов Л.Ю., Наливкин А.В. // Патент на изобретение РФ № 2411530. № 24.11. 2009. 